\title{
Structure and Function of a snoRNP Maturation Complex
}

Shaoxiong Tian ${ }^{1}, \mathrm{Ge} \mathrm{Yu}^{1}$, Huan $\mathrm{He}^{2}$, Peilu Liu${ }^{1}$, Alan G. Marshall11,3, Borries Demeler², Scott M. Stagg1,2*, and Hong $\mathrm{Li}^{1,2 *}$

${ }^{1}$ Department of Chemistry and Biochemistry, Florida State University, Tallahassee, FL 32306, USA.

${ }^{2}$ Institute of Molecular Biophysics, Florida State University, Tallahassee, FL 32306, USA

${ }^{3}$ Ion Cyclotron Resonance Program, The National High Magnetic Field Laboratory, Tallahassee, Florida 32310

${ }^{4}$ Department of Biochemistry, the University of Texas Health Science Center at San Antonio, San Antonio, TX 78229, USA

The Saccharomyces cerevisiae (Sc) R2TP complex affords an Hsp90-mediated scaffolding activity to structurally diverse small ribonucleoprotein particles (snoRNPs). The current lack of structural information on the R2TP complex, however, prevents a mechanistic understanding of this biological process. We have obtained a structural model of the ScR2TP complex made up of two AAA+ ATPases, Rvb1/2p, and two Hsp90 co-chaperone proteins, Tah1p and Pih1p, by a combination of analytical ultracentrifugation, chemical cross-linking, hydrogendeuterium exchange, and electron cryomicroscopy methods. We find that the Pih1pTah1p heterodimer caps the Rvb1/2p heterohexameric ring through its association with the flexible insertion domain of Rvb1/2p and the Pih1p-Tah1p cap is dissociable upon nucleotide binding to Rvb1/2p. Therefore, association of snoRNP intermediates with Pih1p-Tah1p, which is demonstrated for Nop58p here, provides a structural basis for nucleotide-driven binding and release. 